\section{FRI0201 OPHTHALMOLOGICAL FEATURES AT DIAGNOSIS AND IN RECURRENCES IN PATIENTS WITH GIANT CELL ARTERITIS CONFIRMED BY BIOPSY IN A TERTIARY HOSPITAL}

A. De Diego Sola ${ }^{1}$, N. Alcorta Lorenzo ${ }^{1}$, J. A. Valero Jaimes ${ }^{1}$, C. A. Egüés Dubuc $^{1}$, O. Maiz-Alonso ${ }^{1}$, A. C. Blanco Esteban ${ }^{2}$, J. J. Cancio Fanlo', E. Uriarte Isacelaya ${ }^{1}$, J. M. Belzunegui Otano ${ }^{1} .{ }^{1}$ Hospital Universitario Donostia, Rheumatology, Donostia, Spain; ${ }^{2}$ Hospital Universitario Donostia, Ophthalmology, Donostia, Spain

Background: Giant cell arteritis (GCA) presents with visual symptoms in approximately $37 \%^{1}$ of patients at diagnosis. Patients with visual symptoms present, according to some series, lower levels of C-reactive protein (CRP) at diagnosis and associate less headache, fever, cranial nodules or polymyalgia (PM).

Relapses despite glucocorticoid treatment (GC) are frequent (40-60\%). Visual symptoms are very rare in relapses $(0 \%$ in several series). Since most series focus on northern European populations, it is interesting to study the characteristics in our population.

Objectives: To describe visual manifestations in both diagnosis and recurrence of stroke, only in patients diagnosed with temporal artery biopsy. To analyze demographic, clinical and analytical features in patients with and without visual symptoms.

Methods: We retrospectively reviewed the medical records of patients with positive biopsy for GCA at our center from January 2000 through December 2018. Relapse was defined as the appearance of clinical symptoms in a previously asymptomatic patient requiring a dose increase or restart of GCS. Patients with no response to GCS were not included. Qualitative variables are shown with absolute value and percentage and quantitative variables with mean and standard deviation (SD). Kruskal Wallis, Fisher test and Mann-Whitney U test were used for bivariate analysis.

Results: 52 patients were found: 39 women (73.6\%), with an average age at diagnosis of 77.6 years (SD 6.3). At diagnosis, 28 presented visual symptoms (53.84\%): oculomotor paralysis $5(9.61 \%)$, amaurosis fugax $8(15.38 \%)$, blindness $6(11.54 \%)$, decreased visual acuity $8(28.57 \%)$ and other visual symptoms $1(1.92 \%)$. Eleven had monocular symptoms (38\%) and 9 binocular $(32 \%)$, in 10 patients this data was not collected. The symptoms were permanent in 11 (39.2\%) despite GC. Type of visual impairment was: Anterior Ischemic Optic Neuropathy (AION) (12, 42.86\%), impairment of cranial pairs $(5,17.86 \%)$, central retinal artery occlusion $(1,3.57 \%)$, cilioretinal artery occlusion $(1,3.57 \%)$ and Posterior Ischemic Optic Neuropathy (1, 3.57\%).

Of the 52 patients, $17(32.69 \%)$ presented a minimum of one recurrence and 3 $(5.77 \%)$ presented 2 or more. No patient relapsed with visual clinic.

There were no differences among patients with and without visual symptoms in the variables studied (table 1). However, patients with visual symptoms at diagnosis had numerically less PM and cranial nodules.

Table 1. Demographic, clinical and analytical differences between patients with/without visual symptoms

\begin{tabular}{lcc}
\hline Variables & With visual symptoms $(28)$ & Without visual symptoms (24) \\
ESR $>50$ & $17(60.7 \%)$ & $15(62.5 \%)$ \\
ESR $<50$ & $4(14.3 \%)$ & $2(8.33 \%)$ \\
CRP $>10$ & $17(60.7 \%)$ & $13(54.16 \%)$ \\
CRP $<10$ & $3(10.7 \%)$ & $2(8.33 \%)$ \\
Cephalea & $24(85,7 \%)$ & $23(95,8 \%)$ \\
Polymyalgia & $9(32,1 \%)$ & $12(50 \%)$ \\
Constitutional syndrome & $9(32,1 \%)$ & $7(29,1 \%)$ \\
Jaw claudication & $17(60.7 \%)$ & $12(50 \%)$ \\
Cranial nodules & $4(14,3 \%)$ & $8(33,3 \%)$ \\
Sex: Man/Woman & $9 / 19$ & $5 / 19$ \\
Previous PM diagnosis & $4(14,3 \%)$ & $3(12,5 \%)$ \\
\hline
\end{tabular}

Conclusion: In our series, $53 \%$ of the patients presented visual symptoms at diagnosis, a number higher than that described in the literature. It is important to remember that only patients with biopsy-confirmed ACG were included. The most frequent manifestations were AION followed by oculomotor paralysis. A numerically lower percentage of PM and cranial nodules was observed at diagnosis in patients with visual symptoms compared to patients without them, as seen in some series. The absence of visual clinic in recurrences coincides with that reported in the available literature.

References:

[1] Alba M et al. Relapses in Patients With Giant Cell Arteritis Prevalence, Characteristics, and Associated Clinical Findings in a Longitudinally Followed Cohort of 106 Patients. Medicine. 2014;93: 194-201.

Disclosure of Interests: None declared

DOI: 10.1136/annrheumdis-2020-eular.5911

\section{FRI0202 WHAT IS NOT NERVOUS SYSTEM INVOLVEMENT IN BEHÇET SYNDROME: A SURVEY OF PATIENTS WITH BEHÇET SYNDROME REFERRED TO NEUROLOGY}

E. Dincses ${ }^{1}$, E. B. Caliskan ${ }^{2}$, Z. E. Kaya ${ }^{3}$, U. Uygunoglu ${ }^{3}$, M. Tutuncu ${ }^{3}$, S. Saip ${ }^{3}$, A. Siva ${ }^{3}$, M. Melikoglu1, V. Hamuryudan ${ }^{1}$, G. Hatemi ${ }^{1} .{ }^{1} /$ stanbul University - Cerrahpasa, Cerrahpasa Medical Faculty, Department of Internal Medicine, Division of Rheumatology, Behçet's Disease Research Center, Istanbul, Turkey; ${ }^{2}$ Istanbul University - Cerrahpasa, Cerrahpasa Medical Faculty, Istanbul, Turkey; ${ }^{3}$ Istanbul University - Cerrahpasa, Cerrahpasa Medical Faculty, Department of Neurology, Behçet's Disease Research Center, Istanbul, Turkey

Background: Nervous system involvement of Behçet syndrome (neuroBS) is a serious, but infrequent manifestation of Behçet syndrome (BS). Although many BS patients present with signs and symptoms related to the nervous system, several of these are diagnosed with conditions other than neuroBS. The differential diagnosis may be difficult in such patients.

Objectives: To identify conditions mimicking neuroBS among patients with BS and to determine clinical, laboratory and imaging findings that may help the differential diagnosis.

Methods: We retrospectively screened the charts of 500 BS patients who were registered to our clinic between February 2012 and April 2015, to identify those who were referred to neurology at any time during their follow-up. We follow our BS patients in a multidisciplinary clinic and all patients with a sign or symptom related to the nervous system are seen by one of the neurologist members of the clinic. The final diagnoses, as well as presenting signs and symptoms, laboratory and imaging results and results of any other diagnostic modalities were retrieved from patient charts. Patients who did not have a follow-up visit during the last 3 months were invited to the clinic for their final condition. Those who were not able to come, or one of their family members if they were not able to talk, were interviewed on the phone. Results: Among the 500 BS patients who were screened, $116(23 \%)$ were referred to neurology (53 men, 63 women, mean age $32.8( \pm 9.8), 98(84 \%)$ fulfilled ISG criteria). Among these, 29 (5.8\%) were diagnosed with neuroBS, $30(6 \%)$ had other conditions related to the nervous system, $46(9.2 \%)$ were not diagnosed with a nervous system disorder and their symptoms disappeared and $11(2.2 \%)$ were inconclusive and lost to follow-up. Of the 29 patients with neuroBS, 20 had parenchymal involvement, 7 had cerebral venous sinus thrombosis, 1 had concurrent parenchymal involvement and cerebral venous sinus thrombosis and 1 had atypical neuroBS. Of the 30 BS patients who were diagnosed with another nervous system condition, $14(2.8 \%)$ had primary headache syndromes including tension type headache $(n=5)$ and migraine $(n=9), 6(1.2 \%)$ had psychiatric disorders including psychotic disorder $(n=1)$, depression $(n=4)$ and somatization disorder $(n=1)$, the remaining patients had other diagnoses which were entrapment neuropathy $(n=2)$, epilepsy, glial tumor, multiple sclerosis, Meniere's disease, optic neuritis, neuroretinitis, steroid myopathy and polyneuropathy in one patient each. Presentation features such as cerebellar symptoms, motor symptoms, visual problems, altered consciousness, seizure, fever and facial palsy were more common among patients with neuroBS, whereas sensory symptoms and isolated headache were more common among BS patients with other nervous system conditions (Table).

Table. Clinical characteristics of patients with neuroBS versus other diagnoses

\begin{tabular}{|c|c|c|}
\hline Clinical findings at presentation & $\begin{array}{l}\text { BS patients with nerv- } \\
\text { ous system involvement } \\
\text { (neuroBS) ( } n=29)\end{array}$ & $\begin{array}{l}\text { BS patients with } \\
\text { other nervous } \\
\text { system conditions } \\
(n=30)\end{array}$ \\
\hline Only headache & $2(7 \%)$ & $17(56 \%)$ \\
\hline Cerebellar symptoms* & $8(27 \%)$ & $4(13 \%)$ \\
\hline Motor symptoms* & $4(14 \%)$ & $2(7 \%)$ \\
\hline Sensory symptoms* & $3(10 \%)$ & $5(17 \%)$ \\
\hline Visual problems ${ }^{*}$ (diplopia, blurred vision) & $9(31 \%)$ & $1(3 \%)$ \\
\hline Cognitive symptoms ${ }^{\star}$ & $2(7 \%)$ & $2(7 \%)$ \\
\hline $\begin{array}{l}\text { Other* (Alteration of consciousness, } \\
\text { seizure, fever, facial palsy) }\end{array}$ & $9(31 \%)$ & $1(3 \%)$ \\
\hline
\end{tabular}

${ }^{*}$ Accompanying more than 1 symptom/sign

Conclusion: Nervous system conditions other than neuroBS are common in patients with BS who present with nervous system findings. Caution is required to avoid misdiagnosis of these patients as neuroBS.

Disclosure of Interests: Elif Dincses: None declared, E. Buse Caliskan: None declared, Z. Ece Kaya: None declared, Ugur Uygunoglu: None declared, Melih Tutuncu: None declared, Sabahattin Saip: None declared, Aksel Siva: None declared, Melike Melikoglu: None declared, Vedat Hamuryudan Speakers bureau: Pfizer, AbbVie, Amgen, MSD, Novartis, UCB, Gulen Hatemi Grant/ research support from: BMS, Celgene Corporation, Silk Road Therapeutics grant/research support, Consultant of: Bayer, Eli Lilly - consultant, Speakers bureau: AbbVie, Mustafa Nevzat, Novartis, UCB - speaker DOI: 10.1136/annrheumdis-2020-eular.3915 